\title{
HU Protein
}

National Cancer Institute

\section{Source}

National Cancer Institute. HU Protein. NCI Thesaurus. Code C19434.

A DNA-binding and thermostable low-molecular-weight E. coli protein that resembles eukaryotic histones. Acts as a cofactor in transcriptional repression. (from IRB summaries) 